\title{
Two Cases of Lower Body Contouring with a Spiral and Vertical Medial Thigh Lift
}

\author{
Sang Wha Kim, Hyun Ho Han, Je Won Seo, Jung Ho Lee, Deuk Young Oh, Sang Tae Ahn, \\ Jong Won Rhie \\ Department of Plastic and Reconstructive Surgery, Seoul St. Mary's Hospital, The Catholic University of Korea School of Medicine, Seoul, Korea
}

Massive weight loss results in skin excess, leading to an unsatisfying body contour. Various thigh lift procedures can correct flabby skin in the lower leg. We present a lower body contouring technique with a report on two patients. The procedure is determined by the body contour of the patient. As the skin excess in the thigh area tended to appear mostly on the medial side, a vertical medial thigh lift was considered. Moreover, for patients with a pear/ guitar-shaped body contour, we added the spiral thigh lift for skin excess in the buttocks and the lateral thigh area. The extent of tissue to excise was determined by pinching the patient in a standing position. The inferior skin flap was fixed to non-movable tissue, which was helpful for lifting the tissue and preventing the widening of the scar. After the operation, a drain was kept for 3 to 4 days. A compressive garment was used after removing the drain. There were no complications. The patients were discharged 6 to 8 days after the operation. In conclusion, skin excess, especially in the lower body, can be corrected by a thigh lift combining several procedures, varying from person to person.

Keywords Body contouring / Thigh lift / Skin excess
Correspondence: Jong Won Rhie Department of Plastic and Reconstructive Surgery, Seoul St. Mary's Hospital, The Catholic University of Korea School of Medicine, 222 Banpo-daero, Seocho-gu, Seoul 137-040, Korea Tel: +82-2-2258-6142 Fax: +82-2-594-7230

E-mail: rhie@catholic.ac.kr

This article was presented at the 69th congress of the Korean society of the plastic and reconstructive surgeons (name of Meeting) on November 11-13, 2011 (date of presentation)

No potential conflict of interest relevant to this article was reported.

\section{INTRODUCTION}

The westernization of the Korean diet is driving the increase in the number of the obese. Accordingly, various remedies such as dietary control, pharmacotherapy, and surgical treatment have been developed. In particular, surgical operations such as gastrectomy, gastric bypass, and gastric banding are performed on extremely obese patients whose body mass index (BMI) is over $40 \mathrm{~kg} / \mathrm{m}^{2}$ [1]. The problem is that excessive weight loss, caused by surgical treatment, dietary control, or exercise, can result in flabby skin and unsightly contours, there is extreme variation in the extent and exact presentation of this problem from person to person. Thus, it is important to remove flabby skin in the light of individual characteristics. In 2010, we reported one case of belt dermolipectomy to remove flabby skin around the trunk caused by excessive weight loss [2]. This paper introduces the technique of lower body contouring and reports on 2 cases of thigh-lifts performed on the lower extremities including the hips.

\section{CASES}

In 2011, we undertook 2 cases of thigh-lifts performed on the lower extremities. One patient had flabby skin over the medial thighs and a vertical medial thigh-lift was performed. The other patient had excess skin not only on the medial thighs but also on the lateral hip and buttocks. Therefore, a medial vertical thigh- 
lift was performed concurrently with a spiral thigh-lift.

The preoperative design is crucial to an operation, whereby the result is determined. The lower extremity design was made on the patients in standing and supine positions with their lower extremities abducted. First, the pinching test was performed on the whole lower extremities of the patient in a standing position, by which the excision amount was roughly determined. In the case of the spiral thigh-lift, a line was drawn along the inguinal ligament, anterior superior iliac spine, posterior iliac crest, and sacrum via the inferior gluteal fold and perineal-thigh crease, where along the pinching test was performed to determine the excision amount. Then, incision lines were marked with the drawn line as the base line (Fig. 1). In the process, the lower extremities were properly alternated between abduction and adduction in order that the skin of the lateral hip might not be excessively tensed. Next, the medial thigh was designed in a supine position with the lower extremities abducted. A midline was drawn to connect the midpoints of the perineal-thigh crease and that of the medial knee for reference as the skin may droop while the patient is in a supine position. Afterward, the excision amount was determined by the pinching test while the patient was in a supine position with the lower extremities abducted (Fig. 2). The most important point in medial thigh design is not to invade the femoral triangle in order to prevent the injury of the lymphatic system, which could lead to severe complications such as lymphocele. Thus, dissection to the anteromedial thigh should be minimized. If necessary, dissection could be extended

\section{Fig. 1. Design}

The extent of tissue to excise was determined by pinching the patient in a standing position. Marking by the pinching method is seen here. A line was drawn along the inguinal ligament (*), anterior superior iliac spine (\#), posterior iliac crest (+) and sacrum (0) via the inferior gluteal fold and perineal-thigh crease. Then, the pinching test was performed to determine the excision amount and incision lines were marked as the base line.
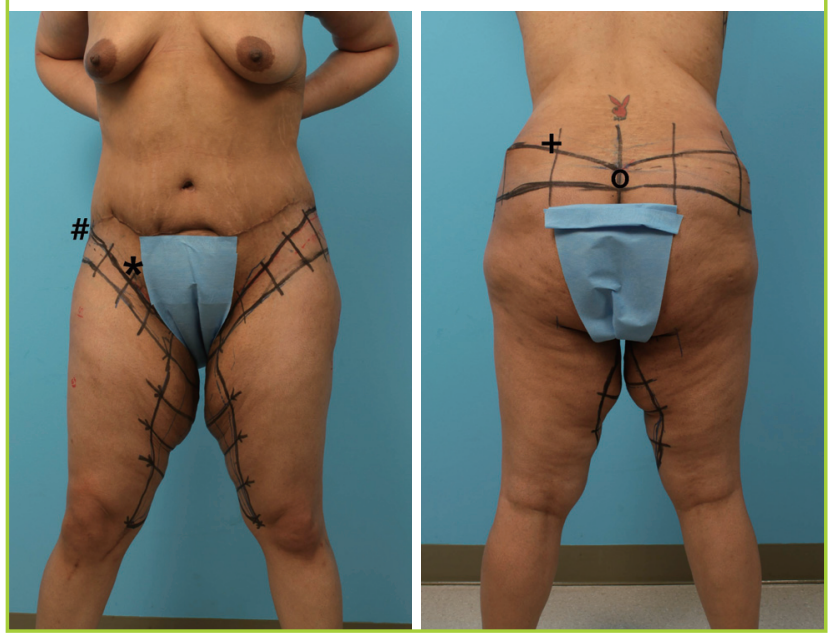

to the posterior aspect from the midline or a design could be made in a bottleneck shape to prevent invasion of the femoral triangle. It is recommended that the femoral triangle be demarcated in the process of preoperative design, which is helpful to prevent invasion during the operation.

The operation was performed in a supine position with the lower extremities abducted. A spreader bar was used to make the patient maintain the frog-leg position. If needed, liposuction is performed beforehand, which was unnecessary in our cases. The medial vertical thigh-lift was performed first. An incision was made on the anterior side, and a dissection was performed toward the posterior thigh through the superficial fat layer, above the superficial fascia system, in order not to injure the major vessels such as the saphenous vein running through the deep fat layer. Considering tension during closure, the excision amount was designed by stages preoperatively and was gradually extended during the course of dissection (Fig. 2). Three to four incisions were made perpendicularly to the incision line dividing the tissue to be excised. It was divided to the proposed incision line and was temporarily fixed by a skin stapler. When it was not so tense, dissection was performed along with staged excision in order to forestall the failure of closure.

In the case of the spiral thigh-lift procedure, a dissection was performed along the inguinal ligament, the anterior superior iliac spine and the lateral hip. Then the patient was turned to the lateral recumbent position and the dissection was extended to the buttock and sacrum. Depending on the circumstances, it is possible to perform the operation after the patient is turned over in the prone position. For the medial thigh-lift procedure, the skin was temporarily fixed by a skin stapler, and the tissue was excised in stages. In the spiral thigh-lift procedure, this requires

Fig. 2. Preoperative design

The excision amount was determined by the pinching test while the patient was in a supine position with the lower extremities abducted and also designed by stages preoperatively and was gradually extended during the course of dissection.

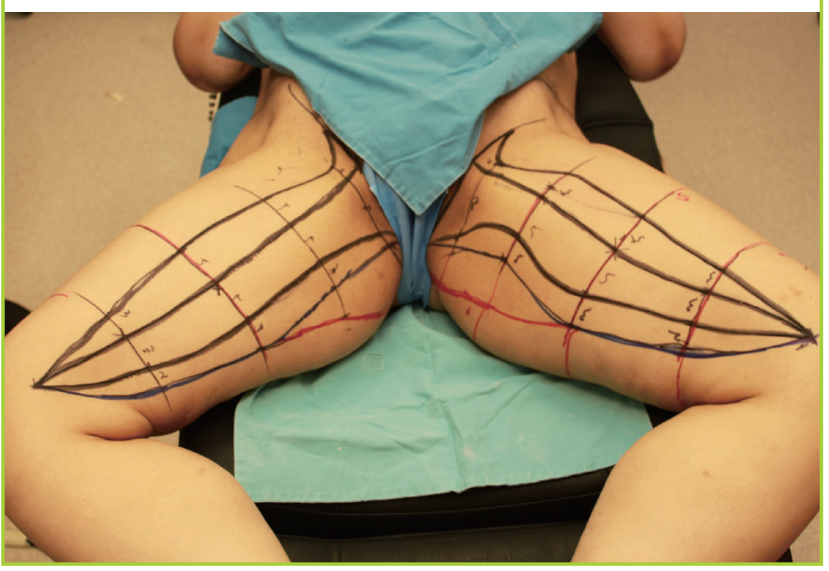


that the inferior skin flap should be fixed to non-movable tissue. Accordingly, the skin flap was fixed to the Colles' fascia in the medial region, to the inguinal ligament in anterior region, and to the periosteum of the anterior superior iliac spine in lateral region, respectively, by the use of $3 / 0$ Vicryl suture. When the medial vertical thigh-lift was performed concurrently with the spiral thigh-lift, there was no need to fix it to the Colles' fascia because the medial vertical thigh-lift alone was enough to lift the skin in the horizontal vector.

An undermining procedure was not performed to prevent hematoma formation and for rapid recovery. The subcutaneous layer was sutured by $3 / 0$ Vicryl suture and $4 / 0$ polydioxanone suture. A surgical drain was inserted. The skin was not sutured but approximated by using surgical tape.

The two operations took 3 and 5 hours. The patients were encouraged to ambulate early on the first postoperative day in order to prevent thromboembolic complications. The drains were removed 3 to 4 days later. In neither cases did complica-

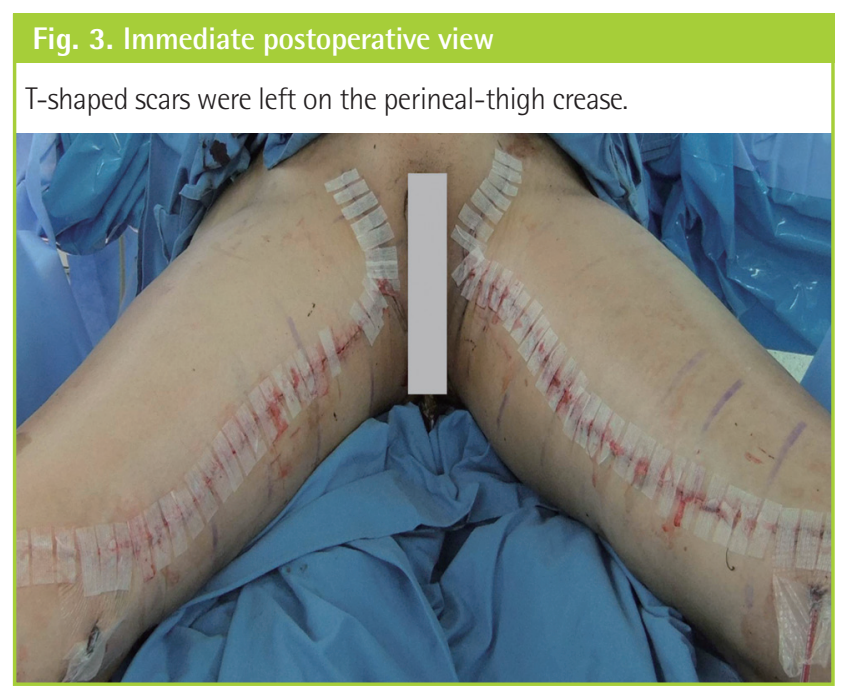

tions such as hematoma formation or wound disruption occur. The patients wore compressive garments after the drains were removed, and were discharged 6 to 8 days later. In the patient who underwent the medial vertical thigh-lift, T-shaped scars were left on the perineal-thigh crease, but did not show when patient was standing (Fig. 3). Follow-up care was conducted for 6 months. Both of them are very satisfied with their final body contours.

\section{Case 1}

A 37-year-old woman had undergone Roux-en-Y bariatric surgery 2 years earlier. Before the operation, she was $182 \mathrm{~cm}$ tall and weighed $192 \mathrm{~kg}$. Her BMI was $58 \mathrm{~kg} / \mathrm{m}^{2}$. Two years later, her weight and BMI were found to be $107 \mathrm{~kg}$ and $32 \mathrm{~kg} / \mathrm{m}^{2}$. However, her skin was flabby all over her body. Therefore, belt dermolipectomy was performed to remove the flabby skin of the trunk. Two months later, reduction mammaplasty and brachioplasty were performed to remove the flabby skin of the chest and upper arms. One year after the secondary operation, flabby skin was observed all over her medial thighs, and further, the flabby skin disturbed her walking. A vertical medial thigh-lift was performed. The quantity of excised tissue was $685 \mathrm{~g}$ from the left thigh and $665 \mathrm{~g}$ from the right thigh. After the three operations, her weight had been reduced by an additional $10 \mathrm{~kg}$.

\section{Case 2}

A 31-year-old woman reduced her weight by $36 \mathrm{~kg}$ by diet and exercise over a one-year period. She was $165 \mathrm{~cm}$ tall, weighed $110 \mathrm{~kg}$, and her BMI was $40 \mathrm{~kg} / \mathrm{m}^{2}$. After she lost weight, her BMI was found to be $27 \mathrm{~kg} / \mathrm{m}^{2}$. First, brachioplasty and abdominoplasty were performed. On physical examinations performed after the primary operation, excess skin was observed not only on the medial thighs but also on the lateral hip and buttocks. It

\section{Fig. 4. Case 2}

(A) Preoperative view. (B) Postoperative 1 month view.
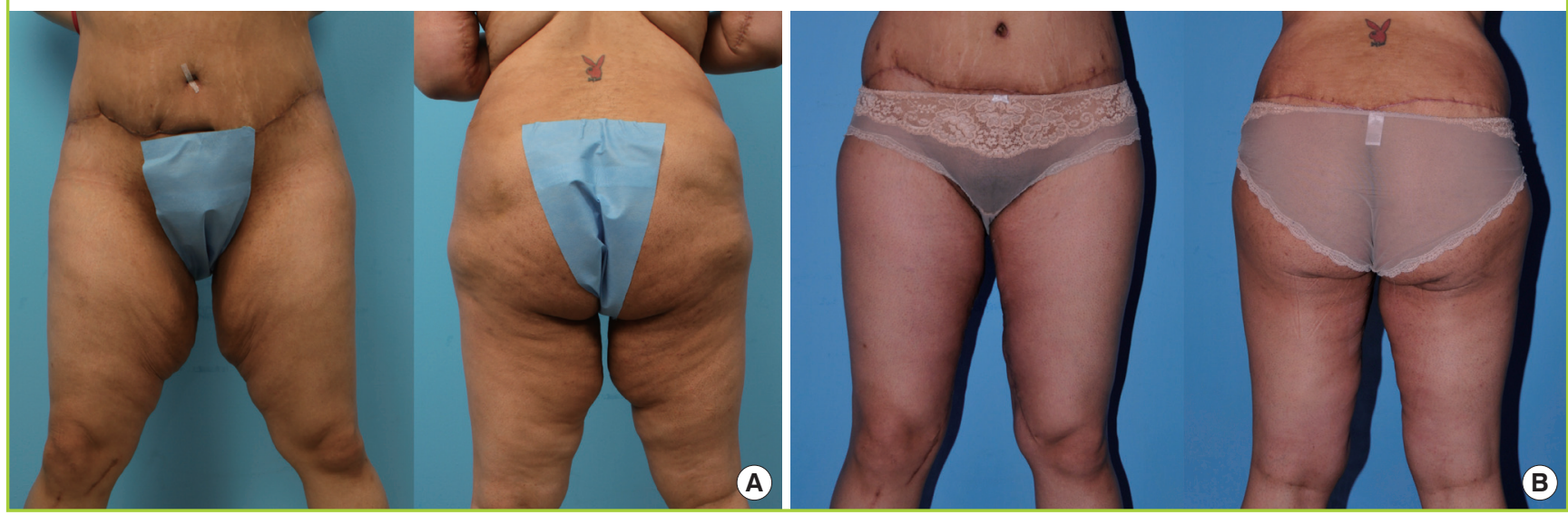
was in the shape of a guitar or pear. Therefore, a medial vertical thigh-lift was performed concurrently with a spiral thigh-lift. Through the secondary operation, 1,344 $\mathrm{g}$ of tissue (642 $\mathrm{g}$ from the left thigh and $702 \mathrm{~g}$ from the right one) were excised (Fig. 4).

\section{DISCUSSION}

Since the thigh-lift was reported by Lewis [3] in 1957, approximately 15,336 cases of thigh-lifts have been performed every year in the US [4]. In Korea, however, it is raely performed. This is because there are few patients who show indications for this procedure. However, the westernization of the Korean diet has increased the obesity rate in Korea, and as a result, body contouring surgeries including thigh-lifts have been becoming more common.

For the medial thigh, the classic Lockwood medial thigh-lift and the vertical medial thigh-lift can be applied [5]. The former is applied in cases where excess skin in a vertical vector is observed on the upper one-third of the medial thigh and where ptosis has occurred. However, realistically, there are few cases where the problem is limited to the upper one-third of the medial thigh. Even if there are such cases, those cases are not severe and most can be solved by liposuction.

The vertical medial thigh-lift is usually applied to cases where the excess skin is in a horizontal vector, especially where excess skin is all over the medial thigh. The effect may persist without the fixation of the dermal layer to the Colle's fascia, because the tension occurs in a horizontal vector during suturing and disperses throughout the medial thigh [5].

In a medial vertical thigh-lift, the first consideration is to preserve the femoral triangle. If the lymphatic system in the femoral triangle is injured, prolonged edema and lymphatic collection may occur. Therefore, the femoral triangle should be demarcated in the process of preoperative design in order that it may not be dissected mistakenly.

The spiral thigh-lift is effective against excess skin and ptosis on the lateral hip (trochanteric area) and buttocks. Although large scars are left, most of them can be covered with underclothes. In addition, it can be performed concurrently with buttock augmentation by use of a gluteal flap [6]. Heretofore, liposuction, belt dermolipectomy and flank-plasty have been substituted for the spiral thigh-lift. The problem is that such operations are inferior to the spiral thigh-lift in the effect against excess skin and ptosis on the lateral hip and buttocks. Furthermore, the problems can recrudesce soon after surgery.

In the case of the spiral thigh-lift, the inferior skin flap is fixed to non-movable tissues such as the Colles' fascia, the inguinal ligament and the periosteum of the anterior superior iliac spine, which makes it possible to lift the buttocks, the lateral hip, and the medial thigh and to prevent recurrence of ptosis shortly after the operation. Moreover, it can prevent scar widening and malformation as well as labium distortion on the medial side $[6,7]$.

We did not perform undermining of the skin flap in these two cases. Excessive undermining increases the risk of hematoma or seroma formation and prolongs hospitalization. This lesson was drawn from our previous case of belt dermolipectomies [2]. It seemed that it would be difficult to fix the inferior skin flap in non-movable tissue without undermining at the time of the treatment to the author. However, in practice, it was easily fixed without tension. Furthermore, the simple procedure without undermining made it possible to shorten the operation time and to ensure rapid recovery and early ambulation of the patients. This, in turn, reduced the risk of a thromboembolic event, which often happens in prolonged operations.

The number of obese people is increasing in Korea. They can suffer from flabby skin after massive weight loss, experiencing many functional and esthetic problems. Skin excess, especially in the lower body, can be corrected by a thigh lift combining several procedures, varying from person to person.

\section{REFERENCES}

1. Shermak MA, Bluebond-Langner R, Chang D. Maintenance of weight loss after body contouring surgery for massive weight loss. Plast Reconstr Surg 2008;121:2114-9.

2. Kim JS, Seo JW, Oh DY, et al. Body contouring of breast and abdomen with belt dermolipectomy after massive weight loss: a case report. J Korean Soc Plast Reconstr Surg 2010; 37:681-6.

3. Lewis JRJr. The thigh lift. J Int Coll Surg 1957;27:330-4.

4. The American Society for Aesthetic Plastic Surgery. Cosmetic Surgery National Data Bank Statistics, 2010 [Internet]. The American Society for Aesthetic Plastic Surgery; c2009 [cited 2012 Jan 3]. Available from: www.surgery.org/media/ statistics.

5. Mathes DW, Kenkel JM. Current concepts in medial thighplasty. Clin Plast Surg 2008;35:151-63.

6. Sozer SO, Agullo FJ, Palladino H. Spiral lift: medial and lateral thigh lift with buttock lift and augmentation. Aesthetic Plast Surg 2008;32:120-5.

7. Kolker AR, Xipoleas GD. The circumferential thigh lift and vertical extension circumferential thigh lift: maximizing aesthetics and safety in lower extremity contouring. Ann Plast Surg 2011;66:452-6. 